Short Communication

\title{
ORAL HEALTH STATUS DURING PREGNANCY IN MANGALORE
}

\author{
Reshma Amin ${ }^{1} \&$ Pushparaja Shetty ${ }^{2}$ \\ ${ }^{1}$ P.G. Student (III Year), ${ }^{2}$ Professor \& HOD, Department of Oral Pathology \& Microbiology \\ A.B. Shetty Memorial Institute of Dental Sciences, Nitte University, Deralakatte \\ M angalore - 575 018, Karnataka, India \\ Correspondence: \\ Reshma Amin \\ P.G. Student (III Year), Department of Oral Pathology \& M icrobiology, A.B. Shetty M emorial Institute of Dental Sciences, \\ Nitte University, Deralakatte, M angalore - 575 018, Karnataka, India. \\ Mobile : +919880544345 E-mail : reshyesh@yahoo.com
}

\begin{abstract}
:
Aim: To assess the oral health status of pregnant and non-pregnant women in the age group of 18 to 35 years in Mangalore.

Materials and Methods: The oral health status of 153 pregnant and 168 non-pregnant women in the designated age group who presented at the outpatient department of a private hospital in Mangalore over a period of 6 months (june 2011-November 2011) was evaluated by various indices including the oral hygiene simplified index (OHI-S), decayed, missing, filled, teeth Index (DM FT) periodontal Index by Russell (PI)

Results : The frequency of occurrence of dental caries in pregnant women was not significantly different from $(p=0.659)$ from that in non-pregnant women, however the $\mathrm{OHI}-\mathrm{S}$ value $(1.031 \pm 0.998)$ was significantly higher $(\mathrm{p}<001)$ in pregnant women than that $(0.592 \pm 0.464)$ in non-pregnant women. Similarly the periodontal index tended to be significantly higher $(\mathrm{P} \triangleleft 0.001)$ in pregnant women $(0.737 \pm 0.476)$ than that in non-pregnant women $(0.378 \pm 0.401)$
\end{abstract}

Conclusion: Indices of oral health tended to be higher in pregnant than in non-pregnant women, suggesting a poorer oral health status in pregnancy in this setting.

Keywords: Oral health, Caries, Indices, Pregnancy.

\section{Introduction :}

The current concept of satisfactory oral health goes beyond possessing healthy teeth, oral health is now known to be integral to general health and essential for well-being (World Health Organization, 2003). While oral health is a key component of overall health and wellbeing of women across their life span, it is particularly important prior to conception and during pregnancy. The physical changes that occur during pregnancy may increase a woman's susceptibility to oral infections, including periodontal disease, and may harm the body's ability to maintain the soft tissues in the mouth. Mild inflammation of the gums

\begin{tabular}{|c|}
\hline Access this article online \\
\hline Quick Response Code \\
\hline
\end{tabular}
(pregnancy gingivitis) is estimated to affect over 30 percent of pregnant women. ${ }^{1}$ Tooth decay may increase during pregnancy due to changes in oral hygiene and diet. Barriers to dental care during pregnancy include persistent myths about the effects of pregnancy on dental health, and concerns for foetal safety during dental treatment. unfornunately patients, physicians, and dentists are also overly cautious, often avoiding treatment of oral health issues during pregnancy. Tooth erosions can result from contact with gastric acids during the 'morning sickness' that may occur during pregnancy. Additionally contributing to the overall health of the woman, and possibly affecting birth outcomes; a mother's oral health status is also important to the health of her child. ${ }^{1}$ Pregnant women with low health literacy have less pregnancy-related knowledge and poorer health behavior. ${ }^{3}$ Numerous metabolic and hormonal changes, considered to be related to fluctuations in estrogen and progesterone levels leading to increased vascular permeability and a decrease in host immunity, favoring increased sensitivity to oral infections. ${ }^{4}$ Therefore improving the oral health of pregnant women will prevent the complications of pregnancy associated dental disease 
and can potentially reduce early childhood caries as well as the preterm and low birth weight deliveries. ${ }^{5}$

Considering all these points the present study was attempted to compare the oral health status (presence of dental caries, gingival and periodontal status ) of pregnant women and non-pregnant women (age range18-35years) in a specific geographic setting (Mangalore, Karnataka India) and to elucidate possible reasons to explain their reluctance to seek dental care during pregnancy.

\section{Materials and Methods:}

The study was done on the pregnant and non-pregnant patients attending the outpatient department of a private hospital in Mangalore. A total of 153 pregnant and 168 non-pregnant women were examined using OHI-S Index, DMFT index, Gingival \& Periodontal index by Russell and the results were tabulated and statistically analysed.

\section{Method :}

In the present study, women between the age group of 18 to 35 years were selected and divided into 2 groups. Group A Pregnant women, Group B Non-Pregnant women. All were examined for oral hygiene status and indices of oral hygiene index simplified (OHI-S), decayed, missing, filled teeth (DMFT) index and Periodontal index (PI) were calculated. The study period was of 6 months (June2011November 2011) on women attending the outpatient department of a private hospital in M angalore, Karnataka, India.

Inclusion and Exclusion criteria: Healthy women ranging in age from 18 to 35years of age coming for antenatal checkup were chosen. Healthy non-pregnant women in the same age group without any systemic disease were chosen for control. Women with systemic diseases like diabetes mellitus, hypertension, and severe anaemia were excluded. The results of various indices were tabulated and statistically analysed.

\section{Results:}

The frequency of occurrence of dental caries in pregnant women was not significantly different from $(p=0.659)$ that in non-pregnant women (Table1), however the OHI-S value
$(1.031 \pm 0.998)$ was significantly higher $(p<001)$ in pregnant women than that $(0.592 \pm 0.464)$ in non-pregnant women (Table2). Similarly the periodontal index tended to be significantly higher $(P<0.001)$ in pregnant women $(0.737 \pm 0.476)$ than that in non-pregnant women (0.378 \pm 0.401$)$ (Table3).

Figl The Bar diagram representing DMFT showing frequency of occurrence of dental caries, incidence of missing, filled teeth in pregnant and non-pregnant women.

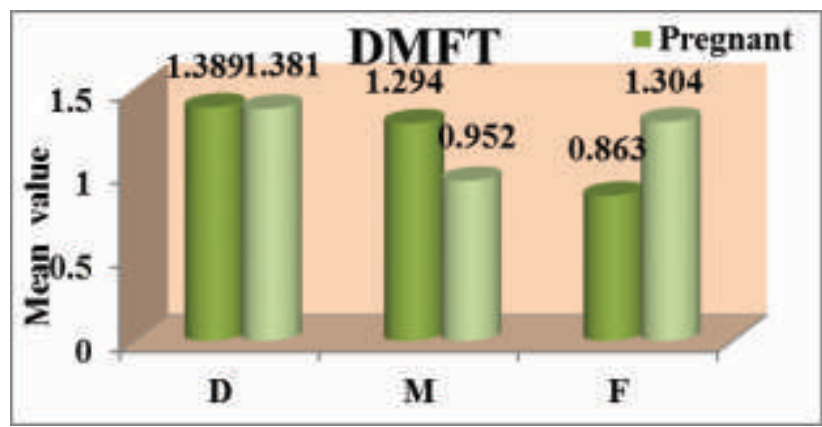

Fig2 The Bar diagram representing oral hygiene simplified index $(\mathrm{OHI}-\mathrm{S})$ showing significantly higher value in pregnant women than that in non-pregnant women.

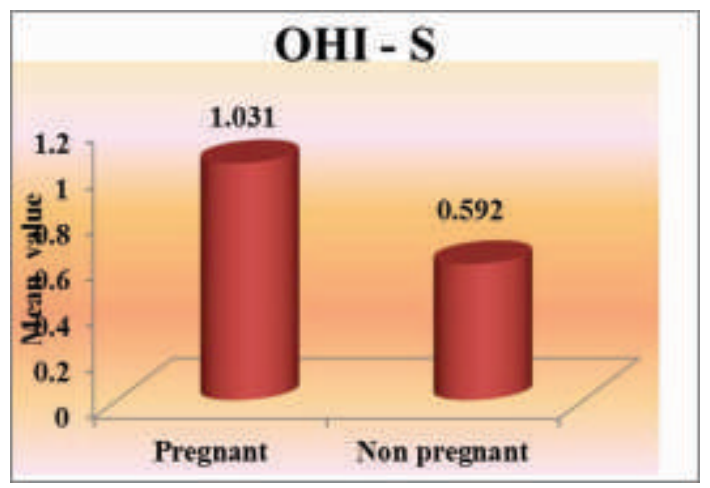

Fig 3 : The Bar diagram representing periodontal index (PI) showing significantly higher value in pregnant women than that in non-pregnant women.

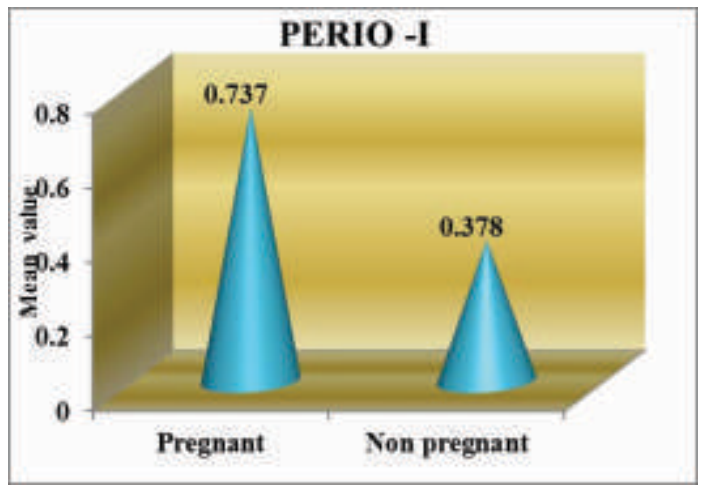


TABLE 1 : Descriptive statistics of DM FT of the study and control group

\begin{tabular}{|ll|r|r|c|c|}
\hline \multicolumn{7}{|c|}{ D M F } \\
\hline & GROUP & $\mathrm{N}$ & M ean & Std. Deviation & Z \\
\hline D & Pregnant & 153 & 1.3856 & 1.90610 & .44200 \\
& Non pregnant & 168 & 1.3810 & 1.74002 & $\mathrm{p}=0.659$ ns \\
\hline M & Pregnant & 153 & 1.2941 & 1.79136 & 1.16200 \\
& Non pregnant & 168 & .9524 & 1.23720 & $\mathrm{p}=0.245 \mathrm{~ns}$ \\
\hline F & Pregnant & 153 & .8627 & 1.74363 & 2.75300 \\
& Non pregnant & 168 & 1.3036 & 1.84020 & $\mathrm{p}=0.006 \mathrm{HS}$ \\
\hline DMF & Pregnant & 153 & 3.5425 & 2.77201 & .47800 \\
& Non pregnant & 168 & 3.6369 & 2.57446 & $\mathrm{p}=0.633 \mathrm{~ns}$ \\
\hline
\end{tabular}

TABLE 2 : Descriptive statistics of OHI-S of the study and control group

\begin{tabular}{|l|r|r|c|c|}
\hline \multicolumn{5}{|c|}{ D M F } \\
\hline \multicolumn{1}{|c|}{ GROUP } & N & M ean & Std. Deviation & Z \\
\hline Pregnant & 152 & 1.0309 & .99803 & 3.62800 \\
Non pregnant & 168 & .5917 & .46435 & $\mathrm{p}<001$ vhs \\
\hline
\end{tabular}

TABLE 3 : Descriptive statistics of PERIO-I of the study and control group

\begin{tabular}{|l|c|c|c|c|}
\hline \multicolumn{5}{|c|}{ D M F } \\
\hline \multicolumn{1}{|c|}{ GROUP } & $\mathrm{N}$ & M ean & Std. Deviation & $\mathrm{Z}$ \\
\hline Pregnant & 153 & .7366 & .47569 & 7.64900 \\
Non pregnant & 168 & .3780 & .40111 & $\mathrm{p}<001$ vhs \\
\hline
\end{tabular}

\section{Discussion:}

The present study was done to to assess the oral health status of pregnant and non-pregnant women in the age group of 18 to 35yrs in Mangalore. Examination of the pregnant women revealed the belief that any sort of treatment during pregnancy is not advisable and thus compounding the existing problems. Immune response, stress and anxiety led to further deterioration, of the already present oral health condition. Pregnancy related problems in the first trimester can result in inadequate attention to oral hygiene and aggravation of the existing oral condition. Correlating the findings of the study showed there was a significant increase in the values of Oral hygiene and Periodontal index, which might lead to an increase in caries on long term.

The present study did not show any increase in caries as compared to the non-pregnant women, the reasons are multiple. One of them could be the background of the patients, it concerned with dietary habits, most of the patients in the present study hailed from lower middle class families who did not show any habit of having snacks between food and a tendency to have food rich in sugar.

The study was not conducted in all trimesters of the same patient and was conducted in a sample size of 153 pregnant women. Considering the fact that long duration is necessary for caries to develop, most women would have developed caries after the delivery, whereas the present study was not extended to the post-partum period and may have been the reason for the present study not showing any rise in the caries incidence as other studies showed. ${ }^{6}$ Furthermore, hormonal changes have long been reported to be associated with changes in oral health during pregnancy. Increase in the rate of both oestrogen metabolism by the gingiva and synthesis of prostaglandins were found to contribute to the gingival changes observed during pregnancy. Alterations in progesterone and estrogen levels have been shown to affect the immune system and both the rate and pattern of collagen production in the gingiva. Both these conditions reduce the body's ability to repair and maintain gingival tissues. So a number of alterations in the oral cavity may become more prevalent during pregnancy. The gingival changes usually resolve within a few months of delivery if local irritants are eliminated. ${ }^{6}$ As observed in this study, both the oral hygiene and gingival index was fairly high.

The results of the present study revealed an association between socio-economic factors such as occupation, education and dental hygiene. Low socio-economic status reportedly suggested inaccessibility to dental clinics and an unawareness to maintain proper oral hygiene. Earlier studies has shown, the risk factors associated with periodontitis during pregnancy are smoking, presence of visible plaque and obesity prior to pregnancy, because periodontal disease is characterized by periods of exacerbation and remission, previous studies showed that the presence of active disease poses a greater risk to pregnancy. ${ }^{7,9}$ Despite their association, studies have demonstrated that pregnancy does not cause periodontitis but rather exacerbates pre-existing periodontal conditions. The present study also shows there is marked increase in 
the association between pregnancy and periodontal condition.

Knowledge or awareness to sustain proper oral hygiene during pregnancy are very crucial for the wellbeing of the pregnant women. There was a definite lack of knowledge on the importance of maintaining oral care in the present study. The belief that oral cavity is not a concerned part in the well-being during pregnancy, was one of the reasons for the observations made in this study. Thus education of women prior to conception and in the ante natal period can prevent most of the dental problems, as well as sustainment of good oral hygiene during and after pregnancy.

\section{References:}

1. Improving Access to Perinatal Oral Health Care: Strategies \& Considerations for Health Plans.NIHCM , July 2010.

2. Silk H, Douglass AB, Douglass JM, Silk L. Oral Health During Pregnancy, Am Fam Physician, 2008;77(8):1139-1144.

3. Hom JM, Lee JY, Divaris K, Baker AD, Vann WF Jr. Oral health literacy and knowledge among patients who are pregnant for the first time.JADA, 2012;143(9):972-980.

4. Suciu BC, M onea A, Cerghizan D, Pop M. Periodontal health in a group of pregnant women. Acta Medica Transilvanica, 2011 June;2(2):292294.

5. Khanna S, M alhotra S. Pregnancy and oral health Forgotten territory revisited J Obstet Gynecol India, 2010;60(2):123-127.

6. Rakchanok N, Amporn D, Yoshida Y, Harun-Or-Rashid M, Sakamoto J dental caries and gingivitis among pregnant and non-pregnant women in chiang mai, Thailand. Nagoyaj. med. Sci, 2010;72:43-50.

7. Bobetsis YA, Offenbacher $S$. Exploring the relationship between periodontal disease and pregnancy Complications. JADA, 2006 October;137.

8. Piscoya MDBV, Ximenes RAA, Silva GM, Jamelli SR, Coutinho SB. Periodontitis-associated risk factors in pregnant women. Clinics, 2012;67(1):27-33.

9. Claas BM, Loschmann LE, Jeffreys M. Self-reported oral health care and access to oral health information among pregnant women in Wellington. NZM J, 201129 July; 124:1339.

\section{CONCLUSION :}

The present study magnifies the problems of managing the oral hygiene among pregnant women when compared to non-pregnant women as it was evident by Oral Hygiene \& Periodontal Index done among both the groups, which were statistically significant, whereas there was no significant rise in the dental caries in pregnant women compared to non-pregnant women. Every pregnant woman should be given instructions to keep up the oral hygiene\& encourage their visit to the dentist, thus establishing a personal sense of responsibility to attain and maintain optimum dental health so as always prevention is better than the cure. 